ERNEST GRLANDU LAWRENEE BERKELEY NATIDNAL LABDRATURY

\title{
Dynamic Aperture Measurements at the Advanced Light Source
}

W. Decking and D. Robin

\section{Advanced Light Source Division}

March 1999 AECEIVED

Presented at the SEP 021999

1999 Particle Accelerator Conference, OSTI New York, NY, March 29-April 2, 1999, and to be published in the Proceedings 


\section{DISCLAIMER}

This document was prepared as an account of work sponsored by the United States Government. While this document is believed to contain correct information, neither the United States Government nor any agency thereof, nor The Regents of the University of California, nor any of their employees, makes any warranty, express or implied, or assumes any legal responsibility for the accuracy, completeness, or usefulness of any information, apparatus, product, or process disclosed, or represents that its use would not infringe privately owned rights. Reference herein to any specific commercial product, process, or service by its trade name, trademark, manufacturer, or otherwise, does not necessarily constitute or imply its endorsement, recommendation, or favoring by the United States Government or any agency thereof, or The Regents of the University of California. The views and opinions of authors expressed herein do not necessarily state or reflect those of the United States Government or any agency thereof, or The Regents of the University of California.

Ernest Orlando Lawrence Berkeley National Laboratory is an equal opportunity employer. 


\section{DISCLAIMER}

Portions of this document may be illegible in electronic image products. Images are produced from the best available original document. 


\title{
Dynamic Aperture Measurements at the Advanced Light SOURCE*
}

\author{
W. Decking and D. Robin \\ Advanced Light Source \\ Lawrence Berkeley National Laboratory \\ University of California \\ Berkeley, CA 94720
}

$3 / 25 / 1999$

"Paper Presented at 1999 Particle Accelerator Conference" New York/New York/U.S.A, March 29th- April 2nd.

*'This work was supported by the Director, Office of Energy Research, Office of Basic Energy Sciences, Materials Sciences Division of the U.S. Department of Energy, under Contract No. DE-AC03-76SF00098 


\title{
DYNAMIC APERTURE MEASUREMENTS AT THE ADVANCED LIGHT SOURCE *
}

\author{
W. Decking ${ }^{\dagger}$, D. Robin, LBNL, Berkeley, USA
}

\section{Abstract}

A large dynamic aperture for a storage ring is of importance for long lifetimes and a high injection efficiency. Measurements of the dynamic aperture of the third generation synchrotron light source Advanced Light Source (ALS) using beam excitation with kicker magnets are presented. The experiments were done for various accelerator conditions, allowing us to investigate the influence of different working points, chromaticities, insertion devices, etc.. The results are compared both with tracking calculations and a simple model for the dynamic aperture yielding good agreements. This gives us confidence in the predictability of the nonlinear accelerator model. This is especially important for future ALS upgrades as well as new storage ring designs.

\section{DEFINITION OF THE APERTURE}

The aperture of an electron storage ring is the maximum transverse and/or longitudinal deviation from the design orbit an electron can experience without being lost. The size of the aperture can effect both injection and lifetime. At the ALS where one injects horizontally offset from the stored beam the on-momentum aperture has to be large enough to accept the injected electron beam. From the point of view of lifetime it is important to have both sufficiently large on-momentum and off-momentum apertures. In particular the lifetime of a low energy, low emittance electron storage ring like the ALS $S^{1}$ is usually given by the scattering of electrons within a bunch (Touschek effect) and/or by elastic and inelastic scattering of electrons with the residual gas.

When electrons scatter within a bunch, they may transfer enough momentum to be outside the momentum aperture of the storage ring. Depending on the dispersion function at the scattering position, scattered electrons start a betatron oscillation in addition to the momentum offset. An electron with a large betatron amplitude usually reaches the off-momentum aperture at smaller momentum deviations. Elastic scattering of electrons with the residual gas excites betatron oscillations. The elastic scattering lifetime is thus proportional to the on-momentum aperture. For more details about aperture measurements using lifetime investigations see [1], [2].

The aperture can be limited by several effects. The linear transverse motion of electrons is limited by the vacuum chamber aperture $x_{v c .}$. In the presence of disper-

\footnotetext{
"This work was supported by the Director, Office of Energy Research, Office of Basic Energy Sciences, Materials Sciences Division, of the U.S. Department of Energy, under Contract No. DE-AC03-76SF00098.

t Now at DESY, Hamburg, Germany.

'The ALS is operated with an energy of $1.5-1.9 \mathrm{GeV}$ and an emittance of $3.5-5.6 \times 10^{-9} \mathrm{radm}$.
}

sion $\eta$ this aperture is reduced by the off-momentum orbit. The invariant physical horizontal aperture is the minimum around the ring of $A_{p h y s, x}(\delta)=\left(x_{v c .}(s)-\eta(s) \delta\right)^{2} / \beta_{x}(s)$ with $\delta=\frac{d P}{P_{0}}$ the relative momentum deviation. Dispersion is usually only present in the horizontal plane leaving the vertical physical aperture $A_{p h y s, y}$ momentum independent. The longitudinal motion of electrons is limited by the height of the rf-bucket provided by the accelerating voltage in the cavity.

The electron motion is also confined by dynamic effects, leading to resonant or chaotic amplitude growth. The border of this motion is called the dynamic aperture $A_{d y n, x}(\delta)$ and depends on the relative momentum deviation. The dynamic aperture is often estimated through tracking calculations.

One can also estimate the dynamic aperture using a simple model in the following way: Electrons are lost when their tune satisfies a resonance condition. From knowing the tune shift terms with amplitude, $\frac{\partial \nu_{y}}{\partial A_{x}}, \frac{\partial \nu_{y}}{\partial A_{y}}$, and momentum deviation, $\frac{\partial \nu_{y}}{\partial \delta}, \frac{\partial^{2} \nu_{y}}{\partial \delta^{2}}$, one can compute the tune shift due to momentum and transverse deviations:

$\Delta \nu_{y}=\frac{\partial \nu_{y}}{\partial A_{x}} A_{d y n, x}+\frac{\partial \nu_{y}}{\partial A_{y}} \kappa A_{d y n, x}+\frac{\partial \nu_{y}}{\partial \delta} \delta+\frac{\partial^{2} \nu_{y}}{\partial \delta^{2}} \delta^{2}+\cdots$

where $\Delta \nu_{y}$ is the distance to the closest 'deadly' resonance, and $\kappa$ is the emittance coupling factor. Knowing the distance to the resonance defines a momentum dependent dynamic aperture.

From equation 1 several ways to increase the dynamic aperture can be seen:

- Increase the distance to the closest 'deadly' resonance by choosing a 'good' working point.

- Decrease the tune shift for large amplitude electrons. This is addressed in the design phase by adequately distributing the nonlinear elements. During operation one can lower the chromaticity $\frac{\partial \nu_{y}}{\partial \delta}$ to small numbers.

- Avoid excitation of resonances, which is achieved in high periodicity machines, where a large number of identical basic cells decreases the number of excitable resonances.

The total aperture is the minimum of all the aperture limitations discussed above. It is often difficult to distinguish clearly between dynamic and physical limits in the measurements. Only good knowledge of the vacuum chamber dimensions, the optical functions and the emittance coupling allows the separation of the two effects. We will thus only use the term dynamic aperture in the following if we can exclude physical limits. 


\section{MEASUREMENTS OF THE APERTURE}

A tool for aperture measurements is a beam kicker magnet. This is a fast magnet which permits the kicking of the beam over a single turn. A criteria for the aperture could be to increase the kick amplitude until the beam is lost. A measurement of this maximum kick amplitude as a function of an artificial aperture limit (horizontal scraper) is shown in figure 1. This measurement serves as a calibration for the kicker voltage. It also shows that at a certain point the kick amplitude is independent of the scraper position. This is when the kicked beam hits another aperture in the ring.

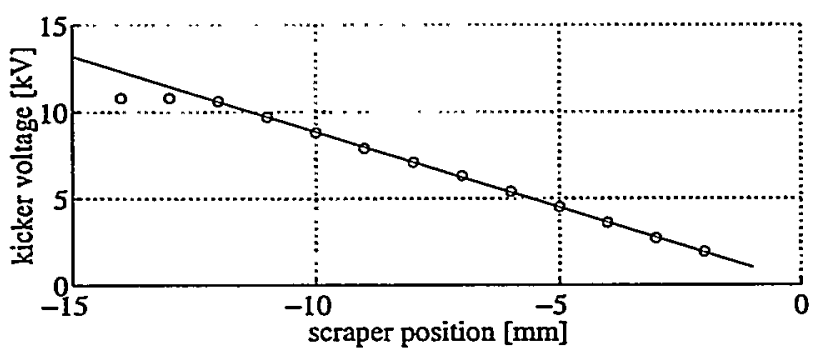

Figure 1: Maximum kicker voltage versus horizontal scraper position.

To measure the aperture for off-momentum electrons, the storage ring rf-frequency is changed to change the storage ring energy. This simulates a 'static' off-momentum situation, as the bunch centroid is not performing synchrotron oscillations. If the main reason for the dynamic aperture is the tune shift with momentum deviation, this 'static' measurement should be sufficient.

Figure 2 shows a measurement of the aperture versus momentum deviation. The measured aperture is asymmetric in momentum due to higher order tune shift terms, which shift the lower momentum electrons faster towards the integer resonance. At a momentum deviation of $\approx$ -0.03 one can see a dip in the curve, which is interpreted as a resonance which is overcome for lower momentum electrons when the tune is shifted beyond this resonance. To estimate the dynamic aperture with synchrotron oscillations, the dynamic aperture is assumed to be the smallest envelope fitting into the measured aperture. With synchrotron oscillations it may be possible for electrons to cross through resonances like the one at $\delta \approx-0.03$ in figure 2 . The synchrotron tune at the ALS is $\nu_{s} \approx 0.008$ or approximately 125 turns per synchrotron oscillation. Therefore the dashed-dotted line, representing the inner envelope, is a somewhat pessimistic approximation of the dynamic aperture including synchrotron oscillations.

Figure 3 shows a comparison of the measured aperture with tracking calculations and with the tune-shift model. Electrons were tracked through the ALS lattice with a sixdimensional symplectic integrator ${ }^{2}$. The following errors

\footnotetext{
${ }^{2}$ The tracking code TRACY2 was used.
}

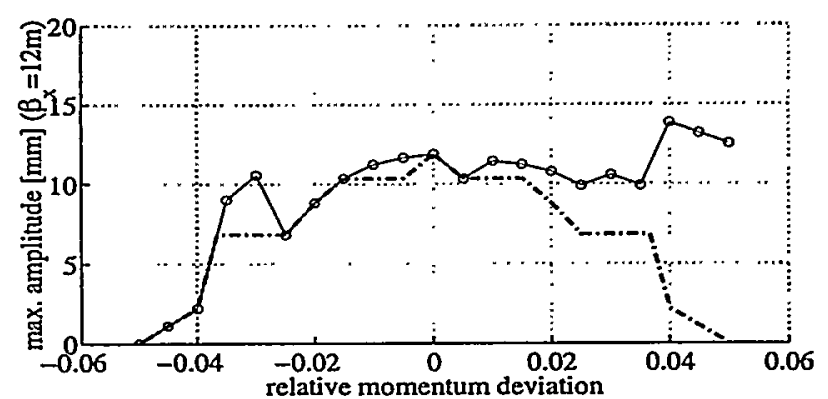

Figure 2: Maximum kick amplitude versus momentum deviation at the following storage ring conditions: $\nu_{x}=$ $0.31, \nu_{y}=0.22, \xi_{x} \approx 0.5, \xi_{y} \approx 0.5$. The solid line is the raw measurement, while the dashed dotted line shows the derived dynamic aperture assuming synchrotron oscillations.

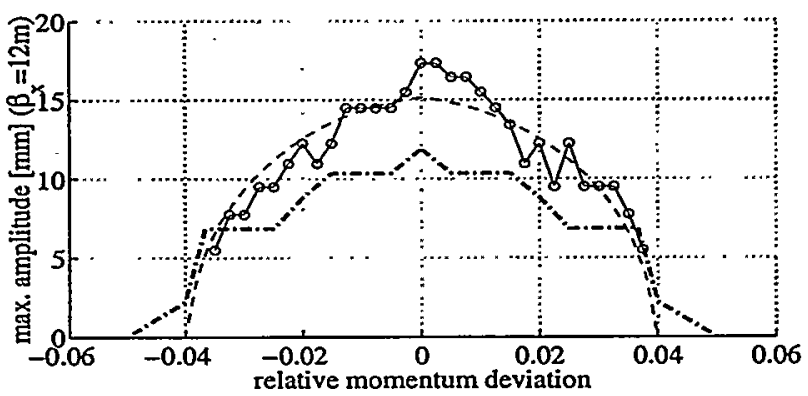

Figure 3: Comparison of measured aperture (dashed-dotted line), tracking calculations (solid line / circles), and tuneshift model with a $\Delta \nu_{y}=-0.14$. The tune was $\nu_{x}=$ $0.31, \nu_{y}=0.22$.

and constraints were included in the model to simulate the realistic machine:

- Physical aperture borders were included in the tracking to prevent electron oscillations outside the realistic vacuum chamber. This is important because large amplitude electrons may perform large, but stable oscillations which would be outside the physical aperture but not lead to a loss of the electron in the tracking.

- Linear field errors are simulated according to the optics measurements done at the ALS with the responsematrix fitting method [3]. This errors lead to a $\beta$-beat and thus a break in periodicity.

- Random skew quadrupole errors were distributed in all quadrupoles of the lattice and adjusted to obtain a $1 \%$ coupling.

- The wiggler (see below) was simulated as a chain of hard edge dipoles obtaining the correct linear focusing and longitudinal dynamics properties.

The tune-shift $\Delta \nu_{y}$ for the tune-shift model was chosen to fit the measured aperture. Measurements, tracking and the tune-shift model agree rather well for large off-momentum electrons. For on-momentum electrons the measurement 
shows smaller horizontal amplitudes. The horizontal physical aperture of the ALS is $\approx 20 \mathrm{~mm}$, thus the measurement (and the tracking) show that the horizontal aperture is limited through dynamic effects.

To investigate the dependence on the tune, the tune is moved towards the integer resonance, parallel to the $\nu_{x}-\nu_{y}$ coupling resonance. Figure 4 shows the aperture for this tune setting, again compared with tracking calculations and the model. The measured dynamic aperture is only slightly smaller than in the previous case (figure 3 ) and the tracking and tune-shift model agree better with the measurements. The integer resonance defines a clear limit for the dynamic aperture. The tracking thus shows the expected behavior (increase of dynamic aperture with distance to the integer resonance) while the measurements indicate that onmomentum electrons are lost at almost the same large transverse amplitudes. Large transverse amplitude particles are for instance more sensitive to additional nonlinear fields, which we have not included in the tracking.

The effect of periodicity breaking in a highly periodic machine can be shown with the insertion of a wiggler ${ }^{3}$ in the ALS lattice. The vertical focusing effect of the wiggler is locally compensated, which leaves a breaking of the ideal 12-fold symmetry. The aperture measurement shows a slight degradation of the dynamic aperture when the wiggler is closed (see figure 5 compared with figure 4). This is in good agreement with tracking calculations.

To show the importance of coupling, an aperture measurement was performed with the skew quad circuits powered. This should decrease the on-momentum dynamic aperture considerably, because electrons with large horizontal amplitudes are coupled in the vertical plane, where they are lost at the small gap vacuum chambers ${ }^{4}$. This situation was also modeled in the tracking. The results are displayed in figure 6 . The agreement between measurement and tracking is good. The tune-shift model fails in this case, because it does not take into account the vertical aperture limit.

\section{CONCLUSION}

The aperture of the ALS was measured with the help of a horizontal kicker magnet. The results of this measurement agree very well with previous measurements using lifetime techniques [1], [2]. The dynamic aperture is also derived from tracking studies and from a rather simple model. The agreement between measurement and tracking is good. The nonlinear accelerator model has to be improved to predict the behavior of large transverse amplitude particles. The simple model can be used to understand the tracking data and to optimize the operating conditions of the ALS. .

\footnotetext{
${ }^{3}$ The wiggler has a peak field of $2 \mathrm{~T}, 0.16 \mathrm{~m}$ period length, and $3 \mathrm{~m}$ total length.

${ }^{4}$ The smallest vacuum chamber gap in the ALS has a full height of $\approx 8 \mathrm{~mm}$ at $\beta_{y}=4 \mathrm{~m}$.
}

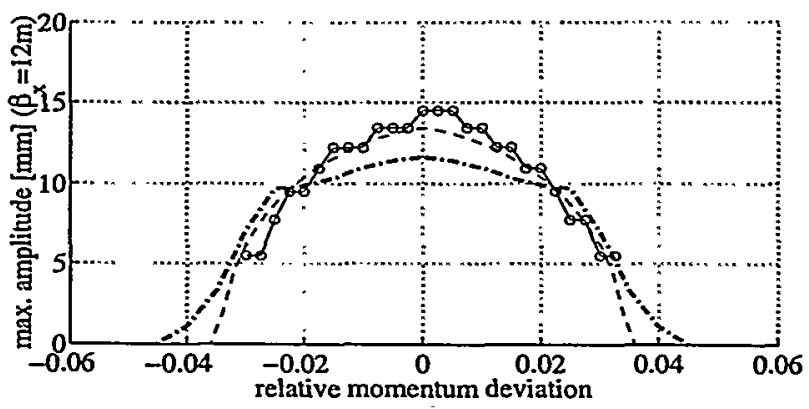

Figure 4: Comparison of measured aperture (dashed-dotted line), tracking calculations (solid line / circles), and tuneshift model with a $\Delta \nu_{y}=-0.11$. The tune was shifted to $\nu_{x}=0.27, \nu_{y}=0.18$.

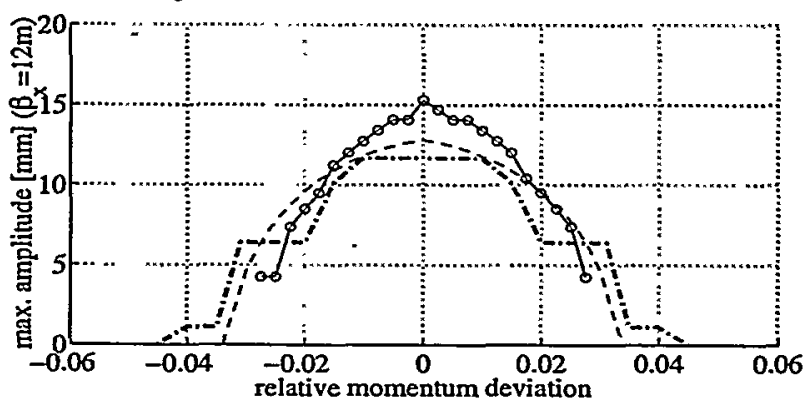

Figure 5: Comparison of measured aperture (dashed-dotted line), tracking calculations (solid line / circles), and tuneshift model with a $\Delta \nu_{y}=-0.1$. The tune was $\nu_{x}=$ $0.27, \nu_{y}=0.18$ and the wiggler was closed.

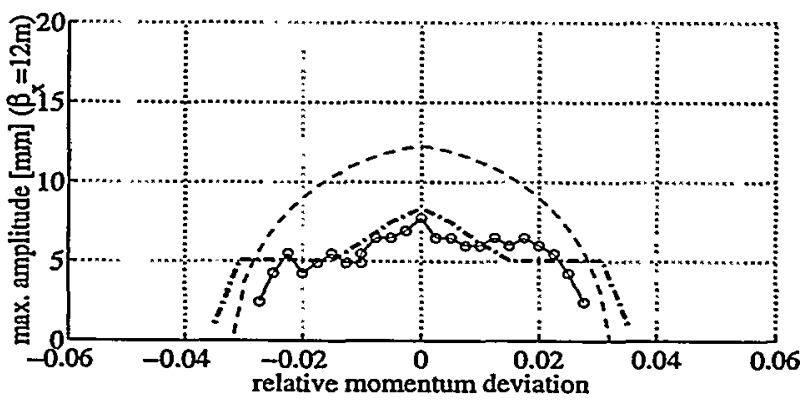

Figure 6: Comparison of measured aperture (dashed-dotted line), tracking calculations (solid line / circles), and tuneshift model with a $\Delta \nu_{y}=-0.09$. The tune was $\nu_{x}=$ $0.27, \nu_{y}=0.18$ and the wiggler was closed. In addition the skew quadrupole circuit was powered.

\section{REFERENCES}

[1] W. Decking et al., "Lifetime Studies at the Advanced Light Source", EPAC98, Stockholm, 1998.

[2] W. Decking, D. Robin, "Momentum Aperture of the Advanced Light Source", EPAC98, Stockholm, 1998, and ICFA Workshop on Nonlinear and Collective Phenomena in Beam Physics, Arcidosso, 1998.

[3] D. Robin et al., "Model Calibration and Symmetry restoration of the Advanced Light Source", EPAC96, Sitges, 1996. 